\title{
A NOTE ON SANTALÓ INEQUALITY FOR THE POLARITY TRANSFORM AND ITS REVERSE
}

\author{
SHIRI ARTSTEIN-AVIDAN AND BOAZ A. SLOMKA
}

(Communicated by Thomas Schlumprecht)

\begin{abstract}
We prove a Santaló and a reverse Santaló inequality for the class consisting of even log-concave functions attaining their maximal value 1 at the origin, also called even geometric log-concave functions. We prove that there exist universal numerical constants $c, C>0$ such that for any even geometric log-concave function $f=e^{-\varphi}$,

$$
c^{n} \cdot\left|B_{2}^{n}\right|^{2} \leq \int_{\mathbb{R}^{n}} e^{-\varphi} \int_{\mathbb{R}^{n}} e^{-\varphi^{\circ}} \leq\left(\left|B_{2}^{n}\right| n !\right)^{2}\left(1+\frac{C}{n}\right)
$$

where $B_{2}^{n}$ is the Euclidean unit ball of $\mathbb{R}^{n}$ and $\varphi^{\circ}$ is the polar function of $\varphi$ (not the Legendre transform!), a transform which was recently rediscovered by Artstein-Avidan and Milman and is defined below. The bounds are sharp up to the optimal constants $c, C$.
\end{abstract}

\section{INTRODUCTION AND MAIN RESULTS}

Let $\mathbb{R}^{n}$ denote the Euclidean $n$-dimensional real space, equipped with the standard scalar product $\langle\cdot, \cdot\rangle$ and the standard Euclidean norm $|x|=\langle x, x\rangle^{1 / 2}$. Denote its unit ball by $B_{2}^{n}$. Let $K \subseteq \mathbb{R}^{n}$ be a convex body (i.e., a compact convex set with non-empty interior). The polar set of $K$ is given by

$$
K^{\circ}=\left\{x \in \mathbb{R}: \sup _{y \in K}\langle x, y\rangle \leq 1\right\} .
$$

The set $K^{\circ}$ is also a convex body, and if $K$ is centrally symmetric, so is its polar $K^{\circ}$. Denote the Lebesgue volume of $K$ by $|K|$. Recall the classical Santaló inequality and its reverse: there exists an absolute constant $c>0$ such that for any dimension $n$ and every centrally symmetric convex body $K \subseteq \mathbb{R}^{n}$,

$$
c^{n} \cdot\left|B_{2}^{n}\right|^{2} \leq|K|\left|K^{\circ}\right| \leq\left|B_{2}^{n}\right|^{2} .
$$

The right hand side is referred to as the Santaló inequality and is due to Santaló [17] (for a simpler proof see [12]). The left hand side inequality is referred to as the reverse Santaló inequality, or the Bourgain-Milman inequality, and is due to J. Bourgain and V. Milman [5]. In fact, the left hand side inequality holds also for convex bodies which are not centrally symmetric. While bounds for the numerical constant $c$ have been improving over the years (see [9, 14]), it is still an open question whether among centrally symmetric convex bodies the cube is a

Received by the editors April 2, 2013 and, in revised form, June 12, 2013.

2010 Mathematics Subject Classification. Primary 52A41, 26A51, 46B10.

Key words and phrases. Santaló and reverse Santaló inequality, polarity transform, log-concave function.

This work was supported by ISF grant No. 247/11. 
minimizer for this product, often called the Mahler product, and whether among general convex bodies a simplex is a minimizer.

Functional versions of the Santaló inequality and its reverse were also established: A function $f: \mathbb{R}^{n} \rightarrow \mathbb{R}$ is said to be log-concave if it is of the form $e^{-\varphi}$ where $\varphi: \mathbb{R}^{n} \rightarrow \mathbb{R} \cup\{\infty\}$ is convex. One interpretation for the "polar" function of $e^{-\varphi}$ may be the function $e^{-\mathcal{L} \varphi}$ where $\mathcal{L}$ is the well-known Legendre transform, given by $\mathcal{L} \varphi(x)=\sup _{y \in \mathbb{R}^{n}}[\langle x, y\rangle-\varphi(y)]$. Note that $e^{-\mathcal{L} \varphi}$ is always log-concave. It was proven that there exists an absolute constant $c>0$ such that for any even log-concave function $f=e^{-\varphi}$ on $\mathbb{R}^{n}$ with $0<\int f<\infty$,

$$
\left(\frac{2 \pi}{c}\right)^{n} \leq \int_{\mathbb{R}^{n}} e^{-\varphi} \cdot \int_{\mathbb{R}^{n}} e^{-\mathcal{L} \varphi} \leq(2 \pi)^{n} .
$$

The right hand side is due to Ball [4]; see also Artstein, Klartag, and Milman [1]. In the latter paper it is actually proved that Gaussians are the only maximizers for this product. For further related results see also 6, 10, 11. The left hand side is due to Klartag and Milman 8]. Note that the Santaló inequality and its reverse for centrally symmetric convex bodies may be recovered by plugging in $f=e^{-\|\cdot\|_{K}^{2} / 2}$, where $\|\cdot\|_{K}$ denotes the norm with unit ball $K$. In the sequel we shall often use $\|\cdot\|_{K}$ to denote the gauge function associated with a convex body $K$, even in the non-symmetric case, so long as the origin belongs to $K$. That is, $\|x\|_{K}=\inf \{r>0: x \in r K\}$. In case the origin belongs to the boundary of $K$, $\|\cdot\|_{K}$ attains the value $+\infty$ whenever the infimum is over an empty set.

In this note, we prove a different functional version of the Santaló inequality and its reverse for an important subclass of (even) log-concave functions, called (even) geometric log-concave functions, consisting of all functions of the form $f=$ $e^{-\varphi}$ where $\varphi$ is a non-negative (even) lower semi-continuous convex function with $\varphi(0)=0$. Such convex functions are respectively referred to as geometric convex functions. A few years ago, Artstein-Avidan and Milman [2,3] proved that on this class, up to trivial obvious modifications, there exist exactly two order-reversing involutions: one is the Legendre transform, and the other, which we shall call here the polarity transform, is defined by $\varphi \mapsto \varphi^{\circ}$ where

$$
\varphi^{\circ}(x)=\sup _{y \in \mathbb{R}^{n}} \frac{\langle x, y\rangle-1}{\varphi(y)} .
$$

Here we agree that $\frac{t}{0}=\infty, \frac{0}{0}=0$ and $\overline{0}=(\overline{\overline{0}})_{+}=0$. The latter is needed only for this definition to work with the identically 0 function. It seems that this transform has appeared only once in the literature (Rockafeller's book [15, p. 136]) before it was rediscovered in [2,3. Among other things, in the latter paper strong reasons were given to explain why this new transform should be considered as the natural extension of the notion of polarity from convex bodies to the class of geometric log-concave functions, and the Legendre transform as the natural extension of the support function.

Since these discoveries, the first named author was asked on several occasions whether a Santaló type inequality holds also for the polarity transform. In this note we answer this question in the affirmative. We prove the following. 
Theorem 1. Let $f=e^{-\varphi}$ be an even geometric log-concave function on $\mathbb{R}^{n}$ such that $0<\int e^{-\varphi}<\infty$. Then

$$
c^{n} \cdot\left|B_{2}^{n}\right|^{2} \leq \int_{\mathbb{R}^{n}} e^{-\varphi} \cdot \int_{\mathbb{R}^{n}} e^{-\varphi^{\circ}} \leq\left(\left|B_{2}^{n}\right| n !\right)^{2}\left(1+\frac{C}{n}\right),
$$

where $c, C>0$ are universal constants independent of $n$ and $\varphi$. The left hand side inequality holds also without the assumption that $\varphi$ is even.

Note that, up to optimal constants $c$ and $C$, one cannot hope for better bounds since for indicators $\mathbb{1}_{K}$ of centrally symmetric convex bodies $K \subseteq \mathbb{R}^{n}$ we recover the Bourgain-Milman lower bound and for $\varphi=e^{-|x|}$ the above product equals $n !^{2} \cdot\left|B_{2}^{n}\right|^{2}$. However, it is far from clear which functions one expects to be the maximizers for this inequality. Moreover, although the product $\int e^{-\varphi} \int e^{-\varphi^{\circ}}$ is invariant under invertible linear transformations, as one can check that $(\varphi \circ A)^{\circ}=$ $\varphi^{\circ} \circ A^{-T}$, it does not imply that this product has maximizers or minimizers. We remark also that the fixed points of the mapping $\varphi \mapsto \varphi^{\circ}$ in dimension 1 have been classified by L. Rotem in [16]. The motivation for classifying these fixed points was that in the convex body case the maximizer for the Mahler product is the unique fixed point of the polarity transform for convex bodies, the Euclidean ball. Thus the maximizer of the product considered in Theorem 1, if it exists, might also be a fixed point, although this was not verified. We do know to show, by symmetrizations, that maximizers, if they exist, must be rotationally invariant.

This note is organized as follows. In Section 2.1 we prove some preliminary facts about log-concave functions, in particular that the class of geometric log-concave functions with (finite) positive integral is closed under the polarity transform, and a simple yet useful generalization of a proposition which appeared in [13] about the connection between level sets of a function and its polar. The proof of Theorem 1 is split into two propositions: the right hand side inequality is proven in Section 3 and the left hand side inequality is proven in Section 4

\section{PRELIMinaRy FACTS}

2.1. Comparing level sets of a function and its polar. Let $f=e^{-\varphi}$ be a geometric log-concave function on $\mathbb{R}^{n}$. Denote the support of $f$ by $\operatorname{supp}(f)=$ $\left\{x: \mathbb{R}^{n}: f(x)>0\right\}$. We adopt the notation of [13] and denote the level sets of $f$ by

$$
\bar{K}_{t}(f):=\left\{x \in \mathbb{R}^{n}: f(x) \geq t\right\},
$$

for any $0<t \leq 1$. Similarly we denote the level sets of $\varphi$ by

$$
\underline{K}_{t}(\varphi):=\left\{x \in \mathbb{R}^{n}: \varphi(x) \leq t\right\},
$$

for any $0 \leq t<\infty$. Note that $\bar{K}_{t}(f)$ is a closed convex set, and also that $\bar{K}_{t}(f)=$ $\underline{K}_{\ln (1 / t)}(\varphi)$. Moreover, note that if $f$ is even, then $\bar{K}_{t}(f)$ are centrally symmetric. Denote the ray emanating from the origin and passing through $x \in \mathbb{R}^{n}$ by $\mathbb{R}_{+} x=$ $\{\alpha x: \alpha \geq 0\}$. The following proposition is a simple yet useful generalization of a proposition of V. Milman and L. Rotem [13, Proposition 11].

Proposition 2. For any geometric convex function $\varphi: \mathbb{R}^{n} \rightarrow[0, \infty]$ and any $s, t>0$ we have that

$$
\left(\underline{K}_{1 / s}(\varphi)\right)^{\circ} \subseteq \underline{K}_{s}\left(\varphi^{\circ}\right) \subseteq(s t+1)\left(\underline{K}_{t}(\varphi)\right)^{\circ} .
$$


Moreover, if $\left(\underline{K}_{1 / s}(\varphi)\right)^{\circ}=\underline{K}_{s}\left(\varphi^{\circ}\right)$ for all $s>0$, then

$$
\varphi= \begin{cases}0, & x \in L, \\ \|x\|_{K}, & x \notin L\end{cases}
$$

for some appropriate, convex sets $K, L$ containing the origin. Equivalently, the restriction of $\varphi$ to every ray $\mathbb{R}_{+} x$ is either linear or a convex indicator $\mathbb{1}_{[0, a]}^{\infty}$. Furthermore, if $\varphi$ is even, then either $\varphi=\mathbb{1}_{K}^{\infty}$ or $\varphi=\|\cdot\|_{K}$ for some convex set $K$.

For $t=1 / s$ Proposition 2 reads $\left(\underline{K}_{1 / s}(\varphi)\right)^{\circ} \subseteq \underline{K}_{s}\left(\varphi^{\circ}\right) \subseteq 2\left(\underline{K}_{1 / s}(\varphi)\right)^{\circ}$, which is exactly [13, Proposition 11]. For the reader's convenience, we provide a geometric proof for both inclusions in Proposition 2 2. To this end, we need to recall the following simple facts about the polarity transform (for proofs see e.g. [3]):

Facts. For any geometric convex functions $\varphi, \psi$ on $\mathbb{R}^{n}$ we have that

1. $\varphi \leq \psi$ if and only if $\psi^{\circ} \leq \varphi^{\circ}$. Also $\left(\varphi^{\circ}\right)^{\circ}=\varphi$.

2. $\max (\varphi, \psi)=\min \left(\varphi^{\circ}, \psi^{\circ}\right)$ where $\min (\varphi, \psi)=\sup \{\phi$ a geometric convex function : $\phi \leq \varphi$ and $\phi \leq \psi\}$.

3. For any convex body $K$ which includes the origin and any $t>0,\left(t\|\cdot\|_{K}\right)^{\circ}=$ $\frac{1}{t}\|\cdot\|_{K^{\circ}}$ and $\left(\mathbb{1}_{K}^{\infty}\right)^{\circ}=\mathbb{1}_{K^{\circ}}^{\infty}$, where $\mathbb{1}_{K}^{\infty}=-\log \left(\mathbb{1}_{K}\right)$.

Proof of Proposition 2. Fix $t>0$. Since $\varphi$ is a geometric convex function, it readily follows that for all $x \in \underline{K}_{t}(\varphi)$,

$$
\mathbb{1}_{\underline{K}_{t}}^{\infty}(x) \leq \varphi(x) \leq t \cdot\|x\|_{\underline{K}_{t}(\varphi)}
$$

and for all $x \notin \underline{K}_{t}(\varphi)$,

$$
t \cdot\|x\|_{\underline{K}_{t}(\varphi)} \leq \varphi(x) \leq \underline{\mathbb{1}}_{\underline{K}_{t}}^{\infty}(x) .
$$

Thus, the following inequality holds:

$$
\min \left(t \cdot\|\cdot\|_{\underline{K}_{t}(\varphi)}, \mathbb{1}_{\underline{K}_{t}(\varphi)}^{\infty}\right) \leq \varphi \leq \max \left(t \cdot\|\cdot\|_{\underline{K}_{t}(\varphi)}, \mathbb{1}_{\underline{K}_{t}(\varphi)}^{\infty}\right) .
$$

Applying the inequality with the polar transform and using Facts 1] 3 yield

$$
\psi_{1}^{t}:=\min \left(\frac{1}{t} \cdot\|\cdot\|_{\underline{K}_{t}^{\circ}(\varphi)}, \mathbb{1}_{\underline{K}_{t}^{\circ}(\varphi)}^{\infty}\right) \leq \varphi^{\circ} \leq \max \left(\frac{1}{t} \cdot\|\cdot\|_{\underline{K}_{t}^{\circ}(\varphi)}, \mathbb{1}_{\underline{K}_{t}^{\circ}(\varphi)}^{\infty}\right)=: \psi_{2}^{t} .
$$

As illustrated in Figure 2.1. we have that $\underline{K}_{s}\left(\varphi^{\circ}\right) \subseteq \underline{K}_{s}\left(\psi_{1}^{t}\right)$ and $\underline{K}_{s}\left(\psi_{1}^{t}\right)=$ $(s t+1) \underline{K}_{t}(\varphi)^{\circ}$ for all $s>0$ and $\underline{K}_{s}\left(\psi_{2}^{t}\right) \subseteq \underline{K}_{s}\left(\varphi^{\circ}\right)$ and $\underline{K}_{s}\left(\psi_{2}^{t}\right)=\underline{K}_{t}^{\circ}(\varphi)$ for all $s \geq \frac{1}{t}$, as desired. Equivalently, one may verify that

$\psi_{1}^{t}(x)=\left\{\begin{array}{ll}0, & x \in \underline{K}_{t}(\varphi)^{\circ}, \\ \frac{1}{t}\|x\|_{\underline{K}_{t}(\varphi)^{\circ}}-\frac{1}{t}, & x \notin \underline{K}_{t}(\varphi)^{\circ},\end{array} \psi_{2}^{t}(x)= \begin{cases}\frac{1}{t}\|x\|_{\underline{K}_{t}(\varphi)^{\circ},} & x \in \underline{K}_{t}(\varphi)^{\circ}, \\ +\infty, & x \notin \underline{K}_{t}(\varphi)^{\circ},\end{cases}\right.$

which together with $\psi_{1}^{t} \leq \varphi^{\circ} \leq \psi_{2}^{t}$ implies the same desired inclusions.

Next, we deal with the equality case of the left hand side inequality; we wish to find all possible forms of a function $\varphi$ that satisfies

$$
\left(\underline{K}_{1 / s}(\varphi)\right)^{\circ}=\underline{K}_{s}\left(\varphi^{\circ}\right) \text { for all } s>0 .
$$




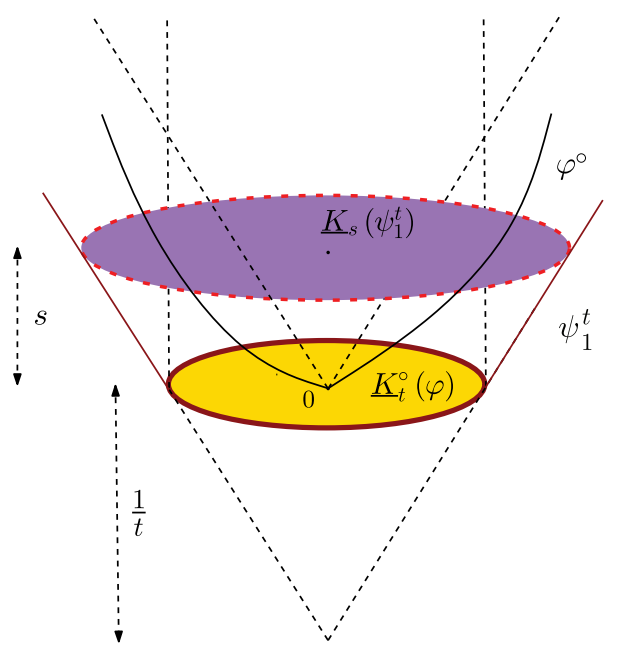

(A) $\underline{K}_{s}^{\circ}(\varphi) \subseteq \frac{s+1 / t}{1 / t} \cdot \underline{K}_{t}\left(\varphi^{\circ}\right)$

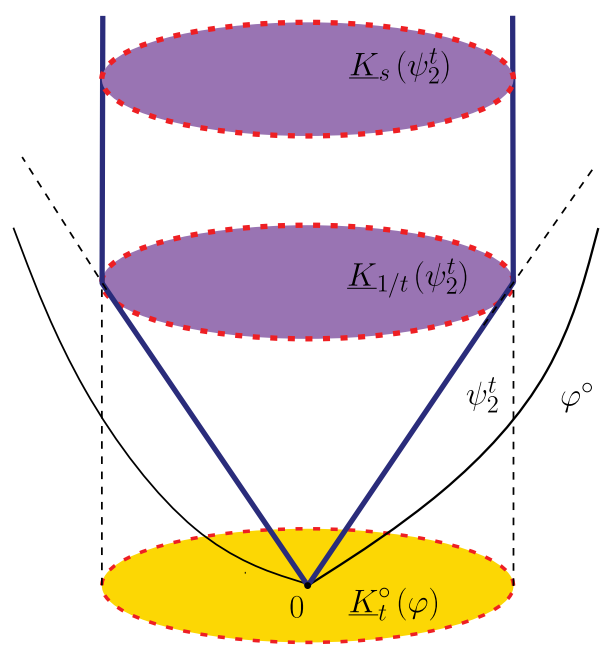

(в) $\underline{K}_{1 / t}(\varphi)^{\circ} \subseteq \underline{K}_{s}\left(\varphi^{\circ}\right)$

Figure 2.1. Comparing level sets of $\varphi$ and $\varphi^{\circ}$ in Proposition 2 , (Color available online.)

Note that, so far, we have that $\varphi^{\circ} \leq \psi_{2}:=\hat{\inf }_{t>0} \psi_{2}^{t}$, or equivalently $\varphi \geq \psi_{2}^{\circ}=$ $\sup _{t>0}\left(\psi_{2}^{t}\right)^{\circ}$. First, we prove that $\psi_{2}^{\circ} \equiv \varphi$ if and only if

$$
\varphi= \begin{cases}\|x\|_{K}, & x \in L, \\ +\infty, & x \notin L,\end{cases}
$$

for some convex sets $K$ and $L$ containing the origin. Indeed, it is not hard to check that such $\varphi$ 's satisfy the desired equality. For the other direction, note that this 
claim is point-wise since

$$
\left(\psi_{2}^{t}\right)^{\circ}(x)= \begin{cases}0, & x \in \underline{K}_{t}(\varphi), \\ t\|x\|_{\underline{K}_{t}(\varphi)}-t, & x \notin \underline{K}_{t}(\varphi),\end{cases}
$$

and so we may assume without loss of generality that the dimension is $n=1$. Let us show that there are no other possible forms of $\varphi$. Assume that $\varphi$ is not of the claimed form. Then without loss of generality (otherwise take $\varphi(-x)$ ), there exist $0<x_{1}<x_{2}$ such that $\varphi^{\prime}\left(x_{1}\right)<\varphi^{\prime}\left(x_{2}\right)$ (and the derivatives exist). Let $0<x_{t}$ denote the point (if it exists) for which $\varphi\left(x_{t}\right)=t$. Then for every $0<t \leq \varphi\left(x_{1}\right)$ we have that

$$
\left(\psi_{2}^{t}\right)^{\circ}\left(x_{2}\right)=\frac{\varphi\left(x_{t}\right)}{x_{t}}\left(x_{2}-x_{t}\right) \leq \frac{\varphi\left(x_{1}\right)}{x_{1}} x_{2}<\varphi\left(x_{2}\right),
$$

for every $\varphi\left(x_{1}\right)<t<\varphi\left(x_{2}\right)$ we have that

$$
\left(\psi_{2}^{t}\right)^{\circ}\left(x_{2}\right)=\frac{\varphi\left(x_{t}\right)}{x_{t}}\left(x_{2}-x_{t}\right) \leq \frac{\varphi\left(x_{2}\right)}{x_{2}}\left(x_{2}-x_{1}\right)<\varphi\left(x_{2}\right)
$$

and for every $t \geq \varphi\left(x_{2}\right)$ we have that $\left(\psi_{2}^{t}\right)^{\circ}\left(x_{2}\right)=0$. Thus,

$$
\psi_{2}^{\circ}\left(x_{2}\right) \leq \sup \left(\frac{\varphi\left(x_{1}\right)}{x_{1}} x_{2}, \frac{\varphi\left(x_{2}\right)}{x_{2}}\left(x_{2}-x_{1}\right)\right)<\varphi\left(x_{2}\right),
$$

as claimed. Note that if $\psi_{2} \neq \varphi^{\circ}$, then $\underline{K}_{s_{0}}\left(\psi_{2}\right) \varsubsetneqq \underline{K}_{s_{0}}\left(\varphi^{\circ}\right)$ for some $s_{0}>0$, and so

$$
\underline{K}_{1 / s_{0}}(\varphi)^{\circ}=\underline{K}_{s_{0}}\left(\psi_{2}^{1 / s_{0}}\right) \subseteq \underline{K}_{s_{0}}\left(\psi_{2}\right) \varsubsetneqq \underline{K}_{s_{0}}\left(\varphi^{\circ}\right) .
$$

Therefore, all geometric convex functions $\varphi$ satisfying (11) are of the form (2) for some convex sets $K$ and $L$ containing the origin. Moreover, note that $\varphi$ satisfies (11) if and only if $\varphi^{\circ}$ satisfies (11) and thus it follows that $\varphi^{\circ}$ is of the form (2) as well. However, we have that

$$
\varphi^{\circ}= \begin{cases}0, & x \in L^{\circ}, \\ \|x\|_{K^{\circ}}\left(1-\frac{1}{\|x\|_{L^{\circ}}}\right), & x \notin L^{\circ},\end{cases}
$$

which means that if $\varphi$ satisfies (11), then it is simultaneously of the form (2) and of the form (3) (with different $K, L$ in each form). It is not hard to check that if $\varphi$ is of both forms, then its restriction to every ray $\mathbb{R}_{+} x$ is either linear or a convex indicator function $\mathbb{1}_{[0, a]}^{\infty}$ (the first form excludes the possibility of attaining 0 on a segment and then linear, and the latter excludes the possibility of attaining linear values on a segment and then attaining $+\infty$ ). Let $\varphi$ be a geometric convex function such that its restriction to every ray $\mathbb{R}_{+} x$ is either linear or a convex indicator. Then $\varphi$ is of both form (2) and form (3). Indeed, define $L=\{\varphi=0\}$ and $K$ by

$$
K \cap \mathbb{R}_{+} x= \begin{cases}\{0\}, & \exists y \in \mathbb{R}_{+} x \varphi(y)=\infty, \\ \{x: \varphi(x) \leq 1\} \cap \mathbb{R}_{+} x, & o / w .\end{cases}
$$

Then one can verify that

$$
\varphi(x)= \begin{cases}0, & x \in L, \\ \|x\|_{K}, & x \notin L\end{cases}
$$


(which is a special case of (3); in every direction $\varphi$ is either linear or a convex indicator), and that $K$ is indeed convex (due to the convexity of $\varphi$ ). Similarly we may define $L^{\prime}=\{\varphi<\infty\}$ and $K^{\prime}$ by

$$
K^{\prime} \cap \mathbb{R}_{+} x= \begin{cases}\{0\}, & \exists y \in \mathbb{R}_{+} x \varphi(y)=\infty, \\ \{x: \varphi(x) \leq 1\}, & o / w .\end{cases}
$$

Again, one can check that $\varphi$ is of the form (2) with $L^{\prime}, K^{\prime}$ in the roles of $L, K$ and that $K^{\prime}$ is convex. Concluding the above, we have that if a geometric convex function $\varphi$ satisfies (11), then its restriction to every ray $\mathbb{R}_{+} x$ is either linear or a convex indicator, and in particular, it is of both form (2) and form (4). Conversely, the restriction of each function of form (4) to every ray is either linear or a convex indicator.

It remains to show that such functions indeed satisfy (11). To this end, let $\varphi$ satisfy (11). Then both $\varphi$ and $\varphi^{\circ}$ are of the form (2). Suppose

$$
\varphi= \begin{cases}\|x\|_{K}, & x \in L, \\ +\infty, & x \notin L,\end{cases}
$$

for appropriate convex sets $K, L$. Then, on the one hand we have that

$$
\underline{K}_{1 / s}(\varphi)=\left\{\|x\|_{K} \leq 1 / s\right\} \cap L=\left(\frac{1}{s} K\right) \cap L .
$$

On the other hand, since $\varphi^{\circ}$ is of the form (2), it follows that

$$
\varphi^{\circ}=\left\{\begin{array}{ll}
0, & x \in L^{\circ}, \\
\|x\|_{K^{\circ}}\left(1-\frac{1}{\|x\|_{L^{\circ}}}\right), & x \notin L^{\circ},
\end{array}= \begin{cases}0, & x \in L^{\circ}, \\
\|x\|_{K^{\circ}}, & x \notin L^{\circ} .\end{cases}\right.
$$

Thus

$$
\underline{K}_{s}\left(\varphi^{\circ}\right)=\operatorname{Conv}\left(\left\{\|x\|_{K^{\circ}} \leq s\right\} \cup L^{\circ}\right)=\operatorname{Conv}\left((s K) \cup L^{\circ}\right)
$$

and so $\underline{K}_{s}\left(\varphi^{\circ}\right)=\underline{K}_{1 / s}(\varphi)^{\circ}$ for all $s>0$ as required. Finally, it is not hard to check that if $\varphi$ is even, then $\varphi$ must be either a norm or a convex indicator of a convex set.

As a consequence, one also gets the following comparison of level sets of a function and the level sets of their Legendre transform. It turns out that this is a special case of [7, Lemma 8]:

Corollary 3. Let $\varphi: \mathbb{R}^{n} \rightarrow[0, \infty]$ be a geometric convex function. Then for every $s>0$ and every $t>0$ we have

$$
s \cdot\left(\underline{K}_{s}(\varphi)\right)^{\circ} \subseteq \underline{K}_{s}(\mathcal{L} \varphi) \subseteq(s+t) \cdot\left(\underline{K}_{t}(\varphi)\right)^{\circ} .
$$

Moreover, if $\left.s \cdot \underline{K}_{s}(\varphi)\right)^{\circ}=\underline{K}_{s}(\mathcal{L} \varphi)$ for all $s>0$, then either $\varphi=\mathbb{1}_{K}^{\infty}$ or $\varphi=\|\cdot\|_{K}$ where $K$ is a closed convex set containing the origin. 
Proof. This follows from the fact, pointed out by V. Milman, that the level sets of $\mathcal{L} \varphi$ and $\varphi^{\circ}$ are connected intimately. Indeed, note that for every $c>0$ we have

$$
\begin{aligned}
\underline{K}_{c}(\mathcal{L} \varphi)=\left\{x \in \mathbb{R}^{n} ;(\mathcal{L} \varphi) \leq c\right\} & =\left\{x \in \mathbb{R}^{n} ; \forall y \in \mathbb{R}^{n},\langle x, y\rangle-\varphi(y) \leq c\right\} \\
& =\left\{x \in \mathbb{R}^{n} ; \forall y \in \mathbb{R}^{n}, \frac{\langle x, y\rangle-c}{\varphi(y)} \leq 1\right\} \\
& =\left\{x \in \mathbb{R}^{n} ; \forall y \in \mathbb{R}^{n}, c \cdot \frac{\left\langle\frac{x}{c}, y\right\rangle-1}{\varphi(y)} \leq 1\right\} \\
& =\left\{c x \in \mathbb{R}^{n} ; \forall y \in \mathbb{R}^{n}, \frac{\langle x, y\rangle-1}{\varphi(y)} \leq \frac{1}{c}\right\} \\
& =c \cdot\left\{x \in \mathbb{R}^{n} ;(\mathcal{A} \varphi) \leq \frac{1}{c}\right\}=c \cdot \underline{K}_{\frac{1}{c}}\left(\varphi^{\circ}\right) .
\end{aligned}
$$

Together with Proposition 2, the proof is thus complete.

2.2. Log-concave functions with finite positive integral. In this section we examine some properties of geometric log-concave functions $f$ satisfying that $0<$ $\int f<\infty$. As later on we will be connecting the integral of such a function to an integral over its level sets, it will be helpful to show that its level sets are convex bodies. This will allow us to apply the classical Santaló (or reverse Santaló) inequality for convex bodies in our setting. The following holds.

Lemma 4. Let $f=e^{-\varphi}$ be a geometric log-concave function on $\mathbb{R}^{n}$. Then $0<$ $\int f<\infty$ if and only if for some open ball $B \subseteq \mathbb{R}^{n}$ and $0<\varepsilon<1, \varepsilon \cdot \mathbb{1}_{B}(x) \leq f(x)$ and for some $r, c>0, f(x) \leq e^{-c|x|}$ for all $|x| \geq r$.

Proof. Assume that $0<\int f<\infty$. The fact that $0<\int f$ obviously implies that $\operatorname{supp}(f)$ is of positive Lebesgue measure. Since $\operatorname{supp}(f)$ is a convex set and $f$ is continuous on its support, there exist $0<\varepsilon<e^{-1}$ and an open ball $B \subseteq \operatorname{supp}(f)$ such that $f(B) \geq \varepsilon$. Denote the unit sphere in $\mathbb{R}^{n}$ by $\mathbb{S}^{n-1}=\left\{\theta \in \mathbb{R}^{n}:|\theta|=1\right\}$. Then for each $\theta \in \mathbb{S}^{n-1}$ there exists $R>0$ such that $f(R \theta) \leq e^{-1}$. Indeed, otherwise we would have that $f(\{\theta r: r \geq 0\})>e^{-1}$ for some $\theta \in \mathbb{S}^{n-1}$ and so, by the log-concavity of $f, f \geq \varepsilon$ on the convex hull of $B$ and the ray $\{\theta r: r \geq 0\}$, which has infinite Lebesgue measure, and so $\int f=\infty$, a contradiction. Hence, by the compactness of $\mathbb{S}^{n-1}$ there exists $r=\min \left\{R>0: f(\theta R) \leq e^{-1}\right.$ for all $\left.\theta \in \mathbb{S}^{n-1}\right\}$, and so for every $x \in \mathbb{R}^{n}$ with $|x| \geq r$,

$$
e^{-1} \geq f\left(\frac{r}{|x|} x\right)=f\left(\frac{r}{|x|} x+\left(1-\frac{r}{|x|}\right) 0\right) \geq f(x)^{r /|x|} f(0)^{1-r /|x|}=f(x)^{r /|x|}
$$

from which it follows that $f \leq e^{-|x| / r}$ for all $|x| \geq r$.

For the opposite direction we have

$$
0<\int \varepsilon \cdot \mathbb{1}_{B}<\int e^{-\varphi}<\int_{|x|<r} e^{-\varphi}+\int_{|x| \geq r} e^{-\varphi} \leq\left|r B_{2}^{n}\right|+\int e^{-c|x|}<\infty .
$$

Remark 5. One may check that the above lemma is equivalent to the fact that a geometric log-concave function $f$ has a finite positive integral if and only if its support is of full dimension and $f$ does not attain the constant value 1 on a whole ray. 
As a consequence of the above lemma we can conclude that the level sets of a geometric log-concave function $f=e^{-\varphi}$ satisfying that $0<\int f<\infty$ are convex bodies and that the class of such even functions is closed under the polarity transform, that is, $0<\int e^{-\varphi^{\circ}}<\infty$ :

Proposition 6. Let $f=e^{-\varphi}$ be a geometric log-concave function on $\mathbb{R}^{n}$, satisfying that $0<\int f<\infty$. Then for all $t>0, \underline{K}_{t}(\varphi)$ and $\underline{K}_{t}\left(\varphi^{\circ}\right)$ are compact convex sets with non-vanishing Lebesgue volume. Moreover, if $f$ is even then $0<\int e^{-\varphi^{\circ}}<\infty$.

Proof. By Lemma 4, $f(x) \leq e^{-c|x|}$ for all $|x| \geq r$ and $\varepsilon \cdot \mathbb{1}_{B} \leq f$ for some $0<\varepsilon<1$ and an open ball $B$. Let $t>0$ such that $x \in \bar{K}_{t}(f)$ for some $|x| \geq r$. Since $f(x) \leq e^{-c|x|}$ for all $|x| \geq r$, it follows that $x \in \bar{K}_{t}\left(e^{-c|\cdot|}\right)$ and so $\left|\bar{K}_{t}(f)\right| \leq$ $\left|\bar{K}_{t}\left(e^{-c|\cdot|}\right)\right|<\infty$. By the same reasoning the last inequality holds for all $s<t$. As $\bar{K}_{t}(f)$ is monotonically decreasing with $t$, we have that $\left|\bar{K}_{t}(f)\right|<\infty$ for all $0<t<1$. In other words, $\left|\underline{K}_{t}(\varphi)\right|<\infty$ for all $t>0$. Since $\varepsilon \cdot \mathbb{1}_{B} \leq f$ it follows that $\bar{K}_{t}\left(\varepsilon \cdot \mathbb{1}_{B}\right) \subseteq \bar{K}_{t}(f)$, and so $0<\left|\bar{K}_{t}\left(\varepsilon \cdot \mathbb{1}_{B}\right)\right| \leq\left|\bar{K}_{t}(f)\right|$, for all $0<t \leq \varepsilon$. In other words, $0<\left|\underline{K}_{t}(\varphi)\right|$ for all $a:=\log (1 / \varepsilon) \leq t$. As $\varphi$ is a geometric convex function, we have that $\varphi(x) \leq a \cdot\|x\|_{\underline{K}_{a}(\varphi)}$ for all $x \in \underline{K}_{a}(\varphi)$, which means that $\underline{K}_{t}\left(a \cdot\|x\|_{\underline{K}_{a}(\varphi)}\right) \subseteq \underline{K}_{t}(\varphi)$ for all $0<t<a$ and hence $0<\left|\underline{K}_{t}(\varphi)\right|$ for all $0<t$. If $f$ is also even, Proposition 2 then implies that

$$
0<\left|\underline{K}_{1 / t}(\varphi)^{\circ}\right| \leq\left|\underline{K}_{t}\left(\varphi^{\circ}\right)\right| \leq 2^{n} \cdot\left|\underline{K}_{1 / t}(\varphi)^{\circ}\right|<\infty
$$

Denote $g(x)=e^{-\varphi^{\circ}(x)}$. Since $0<\left|\underline{K}_{1}\left(\varphi^{\circ}\right)\right|<\infty$ and $\underline{K}_{1}\left(\varphi^{\circ}\right)$ is convex, there exist an open ball $B$ and a radius $r>0$ such that $B \subseteq \underline{K}_{1}\left(\varphi^{\circ}\right) \subseteq r B_{2}^{n}$, and so on the one hand,

$$
\frac{1}{e} \cdot \mathbb{1}_{B} \leq \frac{1}{e} \cdot \mathbb{1}_{\underline{K}_{1}\left(\varphi^{\circ}\right)}=\frac{1}{e} \cdot \mathbb{1}_{\bar{K}_{1 / e}(g)} \leq g=e^{-\varphi^{\circ}}
$$

On the other hand, for some $c>0$ we have that $\varphi^{\circ}(x) \geq\|x\|_{\underline{K}_{1}\left(\varphi^{\circ}\right)} \geq c|x|$ for all $x \notin \underline{K}_{1}\left(\varphi^{\circ}\right)$ and in particular for all $|x| \geq r$. In other words, for all $|x| \geq r$ we have that $e^{-\varphi^{\circ}} \leq e^{-c|x|}$. Thus, Lemma 4 implies that $0<\int e^{-\varphi^{\circ}}<\infty$.

\section{SANTAlÓ INEQUality FOR THE POLARITY TRANSFORM}

Proposition 7. Let $\varphi$ be a geometric even convex function on $\mathbb{R}^{n}$ with $0<\int e^{-\varphi}<$ $\infty$. Then

$$
\int_{\mathbb{R}^{n}} e^{-\varphi} \cdot \int_{\mathbb{R}^{n}} e^{-\varphi^{\circ}} \leq\left(\left|B_{2}^{n}\right| n !\right)^{2}\left(1+\frac{C}{n}\right)
$$

for some universal constant $C>0$ independent of $n$ and $\varphi$.

Proof. We have that

$$
\int_{\mathbb{R}^{n}} e^{-\varphi}=\int_{0}^{1}\left|\bar{K}_{t}\left(e^{-\varphi}\right)\right| d t=\int_{0}^{1}\left|\underline{K}_{\ln (1 / t)}(\varphi)\right| d t=\int_{0}^{\infty} e^{-s} \cdot\left|\underline{K}_{s}(\varphi)\right| d s .
$$


Fix $t>0$. By the convexity of $\varphi$, we have that $\varphi(x) \geq t \cdot\|x\|_{\underline{K}_{t}(\varphi)}$ for all $x \notin \underline{K}_{t}(\varphi)$, from which it follows that $\underline{K}_{s}(\varphi) \subseteq \underline{K}_{s}\left(t\|\cdot\|_{\underline{K}_{t}(\varphi)}\right)$ for all $s>t$. Thus,

$$
\begin{aligned}
\int_{0}^{\infty} e^{-s} \cdot\left|\underline{K}_{s}(\varphi)\right| d s & =\int_{0}^{t} e^{-s} \cdot\left|\underline{K}_{s}(\varphi)\right| d s+\int_{t}^{\infty} e^{-s} \cdot\left|\underline{K}_{s}(\varphi)\right| d s \\
& \leq\left|\underline{K}_{t}(\varphi)\right| \cdot \int_{0}^{t} e^{-s} d s+\int_{t}^{\infty} e^{-s} \cdot\left|\underline{K}_{s}\left(t \cdot\|\cdot\|_{\underline{K}_{t}(\varphi)}\right)\right| d s \\
& =\left|\underline{K}_{t}(\varphi)\right|\left(1-e^{-t}\right)+t^{-n}\left|\underline{K}_{t}(\varphi)\right| \cdot \int_{1}^{\infty} e^{-s} \cdot s^{n} \cdot d s \\
& =\left|\underline{K}_{t}(\varphi)\right|\left[\left(1-e^{-t}\right)+t^{-n} n !\right] .
\end{aligned}
$$

On the other hand, we may apply Proposition 2 and a change of variables to obtain that

$$
\begin{aligned}
\int_{0}^{\infty} e^{-s}\left|\underline{K}_{s}\left(\varphi^{\circ}\right)\right| d s & \leq \int_{0}^{\infty} e^{-s}(s t+1)^{n}\left|\underline{K}_{t}(\varphi)^{\circ}\right| d s \\
& =\left|\underline{K}_{t}(\varphi)^{\circ}\right| \int_{0}^{\infty} e^{-s^{\prime}+\frac{1}{t}} s^{\prime n} t^{n} d s=e^{\frac{1}{t}} t^{n}\left|\underline{K}_{t}(\varphi)^{\circ}\right| n ! .
\end{aligned}
$$

By Proposition 6, $\underline{K}_{t}(\varphi)$ are centrally symmetric convex bodies, and so we may apply the classical Santaló inequality together with the above inequalities to obtain that

$$
\int_{\mathbb{R}^{n}} e^{-\varphi} \cdot \int_{\mathbb{R}^{n}} e^{-\varphi^{\circ}} \leq\left(\left|B_{2}^{n}\right| n !\right)^{2}\left(\frac{\left(1-e^{-t}\right) e^{\frac{1}{t}} t^{n}}{n !}+e^{\frac{1}{t}}\right) \leq\left(\left|B_{2}^{n}\right| n !\right)^{2} e^{\frac{1}{t}}\left(\frac{t^{n}}{n !}+1\right) .
$$

Note that $(n(n+1))^{\frac{1}{n+1}} \leq 3$ and $\frac{n+1}{\sqrt[n+1]{(n+1) !}} \leq e$ for all $n \geq 1$, and so for $t=$ $\sqrt[n+1]{(n-1) !}$ we get that

$e^{\frac{1}{\sqrt[n+1]{(n-1) !}}} \leq 1+e((n-1) !)^{-\frac{1}{n+1}}=1+e(n(n+1))^{\frac{1}{n+1}}((n+1) !)^{-\frac{1}{n+1}} \leq 1+\frac{3 e^{2}}{n+1}$.

Moreover, we have that $e^{\frac{1}{n+1} \sqrt{(n-1) !}} \frac{(\sqrt[n+1]{(n-1) !})^{n}}{n !} \leq \frac{e}{n}$, and so we may conclude that

$$
\int_{\mathbb{R}^{n}} e^{-\varphi} \cdot \int_{\mathbb{R}^{n}} e^{-\varphi^{\circ}} \leq\left(\left|B_{2}^{n}\right| n !\right)^{2}\left(1+\frac{C}{n}\right) .
$$

Remark. As Joseph Lehec suggested to the authors after reading a first draft of this note, one may alternatively use Ball's argument (4]; see also [6]) to prove essentially the same inequality. Namely, the fact that for every geometric convex function,

$$
\frac{\varphi(x)+\varphi^{\circ}(y)}{2} \geq \sqrt{\varphi(x) \varphi^{\circ}(y)}=\sqrt{\sup _{z \in \mathbb{R}^{n}} \varphi(x) \cdot \frac{\langle z, y\rangle-1}{\varphi(z)}} \geq \sqrt{(\langle x, y\rangle-1)_{+}}
$$

implies, by Ball's argument, that

$$
\int_{\mathbb{R}^{n}} e^{-\varphi} \int_{\mathbb{R}^{n}} e^{-\varphi^{\circ}} \leq\left(\int_{\mathbb{R}^{n}} e^{-\sqrt{\left(|x|^{2}-1\right)_{+}}}\right)^{2}
$$

which is qualitatively the same as our bound. 


\section{REVERSE SANTAló INEQUALITy FOR THE POLARITy TRANSFORM}

Proposition 8. Let $\varphi$ be a geometric convex function on $\mathbb{R}^{n}$ with $0<\int e^{-\varphi}<\infty$. Then

$$
0.7 \cdot c^{n} \cdot\left|B_{2}^{n}\right|^{2} \leq \int_{\mathbb{R}^{n}} e^{-\varphi} \cdot \int_{\mathbb{R}^{n}} e^{-\varphi^{\circ}}
$$

where $c>0$ is the best constant for the classical reverse Santalo inequality for convex bodies.

Proof. As in the proof of Proposition 7, we have that $\int_{\mathbb{R}^{n}} e^{-\varphi}=\int_{0}^{\infty} e^{-s} \cdot\left|\underline{K}_{s}(\varphi)\right| d s$. By Proposition 2. Cauchy-Schwarz inequality, and the classical reverse Santaló inequality, we obtain that

$$
\begin{aligned}
\int_{\mathbb{R}^{n}} e^{-\varphi} \cdot \int_{\mathbb{R}^{n}} e^{-\varphi^{\circ}} & =\int_{0}^{\infty} e^{-s} \cdot\left|\underline{K}_{s}(\varphi)\right| d s \cdot \int_{0}^{\infty} e^{-u} \cdot\left|\underline{K}_{u}\left(\varphi^{\circ}\right)\right| d u \\
& \geq \int_{0}^{\infty} e^{-s} \cdot\left|\underline{K}_{s}(\varphi)\right| d s \cdot \int_{0}^{\infty} e^{-u} \cdot\left|\underline{K}_{1 / u}(\varphi)^{\circ}\right| d u \\
& =\int_{0}^{\infty} e^{-s} \cdot\left|\underline{K}_{s}(\varphi)\right| d s \cdot \int_{0}^{\infty} \frac{e^{-1 / t}}{t^{2}} \cdot\left|\underline{K}_{t}(\varphi)^{\circ}\right| d t \\
& \geq\left(\int_{0}^{\infty} \sqrt{e^{-s} \cdot\left|\underline{K}_{s}(\varphi)\right|} \cdot \sqrt{\frac{e^{-1 / s}}{s^{2}}} \cdot\left|\underline{K}_{s}(\varphi)^{\circ}\right| d s\right)^{2} \\
& \geq c^{n} \cdot\left|B_{2}^{n}\right|^{2} \cdot\left(\int_{0}^{\infty} \frac{e^{-s / 2-1 /(2 s)}}{s} d s\right)^{2} \\
& \geq a \cdot c^{n} \cdot\left|B_{2}^{n}\right|^{2},
\end{aligned}
$$

where

$$
a=\left(\int_{0}^{\infty} \frac{e^{-s / 2-1 /(2 s)}}{s} d s\right)^{2} \geq 0.7
$$

may be computed numerically.

\section{ACKNOWLEDGMENTS}

The authors would like to thank Joseph Lehec for his insightful remarks. The authors would also like to thank Matthieu Fradelizi for referring them to the paper [7. Finally, the authors wish to thank Keshet Einhorn and Liran Rotem for their useful comments and corrections.

\section{References}

[1] S. Artstein-Avidan, B. Klartag, and V. Milman, The Santaló point of a function, and a functional form of the Santaló inequality, Mathematika 51 (2004), no. 1-2, 33-48 (2005), DOI 10.1112/S0025579300015497. MR2220210(2007a:52008)

[2] S. Artstein-Avidan and V. Milman, A characterization of the support map, Adv. Math. 223 (2010), no. 1, 379-391, DOI 10.1016/j.aim.2009.07.020. MR2563222 (2011g:52002)

[3] S. Artstein-Avidan and V. Milman, Hidden structures in the class of convex functions and a new duality transform, J. Eur. Math. Soc. (JEMS) 13 (2011), no. 4, 975-1004, DOI 10.4171/JEMS/273. MR2800482 (2012h:49069)

[4] K. M. Ball, PhD dissertation, Cambridge.

[5] J. Bourgain and V. D. Milman, New volume ratio properties for convex symmetric bodies in $\mathbf{R}^{n}$, Invent. Math. 88 (1987), no. 2, 319-340, DOI 10.1007/BF01388911. MR880954 (88f:52013) 
[6] M. Fradelizi and M. Meyer, Some functional forms of Blaschke-Santaló inequality, Math. Z. 256 (2007), no. 2, 379-395, DOI 10.1007/s00209-006-0078-z. MR2289879 (2008c:52013)

[7] M. Fradelizi and M. Meyer, Increasing functions and inverse Santaló inequality for unconditional functions, Positivity 12 (2008), no. 3, 407-420, DOI 10.1007/s11117-007-2145-z. MR2421143(2009e:26025)

[8] B. Klartag and V. D. Milman, Geometry of log-concave functions and measures, Geom. Dedicata 112 (2005), 169-182, DOI 10.1007/s10711-004-2462-3. MR2163897(2006d:52004)

[9] G. Kuperberg, From the Mahler conjecture to Gauss linking integrals, Geom. Funct. Anal. 18 (2008), no. 3, 870-892, DOI 10.1007/s00039-008-0669-4. MR2438998(2009i:52005)

[10] J. Lehec, A direct proof of the functional Santaló inequality (English, with English and French summaries), C. R. Math. Acad. Sci. Paris 347 (2009), no. 1-2, 55-58, DOI 10.1016/j.crma.2008.11.015. MR.2536749(2010h:26027)

[11] J. Lehec, Partitions and functional Santaló inequalities, Arch. Math. (Basel) 92 (2009), no. 1, 89-94, DOI 10.1007/s00013-008-3014-0. MR2471991 (2010a:39017)

[12] M. Meyer and A. Pajor, On the Blaschke-Santaló inequality, Arch. Math. (Basel) 55 (1990), no. 1, 82-93, DOI 10.1007/BF01199119. MR1059519 (92b:52013)

[13] V. Milman and L. Rotem, Mixed integrals and related inequalities, J. Funct. Anal. 264 (2013), no. 2, 570-604, DOI 10.1016/j.jfa.2012.10.019. MR.2997392

[14] F. Nazarov, The Hörmander proof of the Bourgain-Milman theorem, Geometric aspects of functional analysis, Lecture Notes in Math., vol. 2050, Springer, Heidelberg, 2012, pp. 335343, DOI 10.1007/978-3-642-29849-3_20. MR2985302

[15] R. Tyrrell Rockafellar, Convex analysis, Princeton Mathematical Series, No. 28, Princeton University Press, Princeton, N.J., 1970. MR.0274683 (43 \#445)

[16] L. Rotem, Characterization of self-polar convex functions, Bull. Sci. Math. 136 (2012), no. 7, 831-838, DOI 10.1016/j.bulsci.2012.03.003. MR2972564

[17] L. A. Santaló, An affine invariant for convex bodies of n-dimensional space (Spanish), Portugaliae Math. 8 (1949), 155-161. MR0039293(12,526f)

School of Mathematical Science, Tel-Aviv University, Ramat Aviv, Tel Aviv, 69978, ISRAEL

E-mail address: shiri@post.tau.ac.il

School of Mathematical Science, Tel-Aviv University, Ramat Aviv, Tel Aviv, 69978, ISRAEL

E-mail address: boazslom@post.tau.ac.il 